\title{
Determining the Optimal Vaccination Schedule for Herpes Zoster: a Cost-Effectiveness Analysis
}

\author{
Phuc Le, PhD, MPH and Michael B. Rothberg, MD, MPH \\ Center for Value-Based Care Research, Medicine Institute, Cleveland Clinic, Cleveland, OH, USA.
}

\begin{abstract}
BACKGROUND: The Advisory Committee on Immunization Practices recommends a single dose of herpes zoster (HZ) vaccine in persons aged 60 years or older, but the efficacy decreases to zero after approximately 10 years. A booster dose administered after 10 years might extend protection, but the cost-effectiveness of a booster strategy has not been examined.
\end{abstract}

OBJECTIVE: We aimed to determine the optimal schedule for $\mathrm{HZ}$ vaccine

DESIGN: We built a Markov model to follow patients over their lifetime. From the societal perspective, we compared costs and quality-adjusted life years (QALYs) saved of 11 strategies to start and repeat $\mathrm{HZ}$ vaccine at different ages. SUBJECTS: Adults aged 60 years.

INTERVENTION: $\mathrm{HZ}$ vaccine.

MAIN MEASURES: Costs, quality-adjusted life years (GALYs), and incremental costs per GALY saved.

KEY RESULTS: At a \$100,000/QALY threshold, "vaccination at 70 plus one booster" was the most cost-effective strategy, with an incremental cost-effectiveness ratio (ICER) of $\$ 36,648 /$ QALY. "Vaccination at 60 plus two boosters" was more effective, but had an ICER of $\$ 153,734 /$ QALY. In deterministic sensitivity analysis, "vaccination at 60 plus two boosters" cost $<\$ 100,000 /$ QALY if compliance rate was $>67 \%$ or vaccine cost was $<$ \$156 per dose. In probabilistic sensitivity analysis, "vaccination at 70 plus one booster" was preferred at a willingness-to-pay of up to $\$ 135,000 /$ QALY.

CONCLUSIONS: Under current assumptions, initiating $\mathrm{HZ}$ vaccine at age 70 years with one booster dose 10 years later appears optimal. Future data regarding compliance with or efficacy of a booster could affect these conclusions.

KEY WORDS: herpes zoster; cost-effectiveness; booster.

J Gen Intern Med 32(2): 159-67

DOI: $10.1007 / \mathrm{s} 11606-016-3844-6$

(C) Society of General Internal Medicine 2016

\section{INTRODUCTION}

Herpes zoster (HZ) is a painful dermatomal vesicular rash afflicting almost one in three adults. ${ }^{1}$ Of the approximately one million Americans who experience $\mathrm{HZ}$ annually, 8-32 \%

Electronic supplementary material The online version of this article (doi:10.1007/s11606-016-3844-6) contains supplementary material, which is available to authorized users.

Received April 7, 2016

Revised July 11, 2016

Accepted July 29, 2016

Published online October 14, 2016 develop postherpetic neuralgia (PHN), an often debilitating pain persisting greater than 3 months. ${ }^{2}$ The frequency and severity of $\mathrm{HZ}$ and PHN increase with age, especially after 50 years. ${ }^{1}$ Because antiviral therapy has limited effectiveness, efforts have focused on prevention. ${ }^{3}$

The live attenuated Oka strain VZV vaccine reduces the incidence of $\mathrm{HZ}, \mathrm{PHN}$ and their associated burden-of-illness (BOI) among people $\geq 50$ years of age, ${ }^{4-7}$ but the Advisory Committee on Immunization Practices (ACIP) recommends the vaccine only for adults aged $\geq 60$ years, ${ }^{8}$ in part because the vaccine appears not to be cost-effective below this age. ${ }^{9}$ For adults younger than 60 years, this policy is a de facto recommendation to be vaccinated on turning 60 years. Because vaccine efficacy declines to zero over approximately 10 years, ${ }^{4}$ the current recommendation could leave adults lacking protection after age 70, when PHN is most prevalent. A booster dose administered after 10 years might extend protection, but the cost-effectiveness of a booster strategy has not been examined.

Recently, a clinical trial assessed the safety and immunogenicity of a 10 -year booster dose administered to adults aged $\geq 70$ years and found it to be safe and to produce antibody responses similar to those of patients vaccinated for the first time. ${ }^{10}$ The discovery of waning immunity and the possibility of one or more booster doses add complexity to the decision about when to vaccinate. Early vaccination with one or more boosters should be most effective, but could be expensive. Waiting to vaccinate until rates of $\mathrm{HZ}$ and $\mathrm{PHN}$ begin to rise might prevent a substantial proportion of disease at lower cost. We used a Markov decision model to examine the costeffectiveness of competing vaccination schedules incorporating new data on vaccine persistence, ${ }^{4}$ and safety and immunogenicity of a booster dose. ${ }^{10}$

\section{METHODS}

We updated a previously published Markov model ${ }^{9,}{ }^{11}$ (online Appendix Figure 1) and compared the cost-effectiveness of 11 strategies: no vaccination, one-time vaccination at 5 -year intervals from 60 to 80 years, vaccination at 5-year intervals from 60 to 75 years with a booster after 10 years, and vaccination at 60 years with two boosters 10 years apart. The entire cohort entered the model at age 60 and was followed for a lifelong time horizon (or to age 120) with a Markov-cycle length of 1 year. People could receive no vaccine or one of the 
Table 1. Model Inputs

\begin{tabular}{|c|c|c|c|c|}
\hline Variable & Baseline value & $\begin{array}{l}\text { Range for one-way } \\
\text { sensitivity analysis }\end{array}$ & PSA Distribution & Reference \\
\hline $\mathrm{HZ}$ incidence/1000 person-years ${ }^{\mathrm{a}}$ & & & & 12,13 \\
\hline \multicolumn{5}{|l|}{ Male } \\
\hline Age $60-69$ & 8.9 & $8.4-9.3$ & Beta & \\
\hline Age $70-79$ & 11.3 & $10.7-11.9$ & & \\
\hline Age $\geq 80$ & 12.2 & $11.2-13.3$ & $\S$ & \\
\hline \multicolumn{5}{|l|}{ Female } \\
\hline Age 60-69 & 12.5 & $12-12.9$ & Beta & \\
\hline Age $70-79$ & 15.1 & $14.4-15.7$ & $\S$ & \\
\hline Age $\geq 80$ & 16.5 & $15.6-17.6$ & $\S$ & \\
\hline Complications & & & & \\
\hline \multicolumn{4}{|l|}{ PHN given $\mathrm{HZ}$} & 5 \\
\hline Age $60-69$ & 0.069 & $0.042-0.096$ & Beta & \\
\hline Age $\geq 70$ & 0.185 & $0.142-0.228$ & & \\
\hline PHN from 6 to 12 months & 0.215 & $0.188-0.247$ & Beta & 14 \\
\hline \multicolumn{4}{|l|}{$\mathrm{PHN} \geq 12$ months } & 15 \\
\hline Age $<70$ & 0.31 & $0.06-0.56$ & Beta & \\
\hline Age $\geq 70$ & 0.52 & $0.34-0.70$ & & \\
\hline Any ophthalmic complications & 0.022 & $0.012-0.032$ & Beta & 11 \\
\hline Monocular blindness, given ophthalmic complications & 0.039 & $0.011-0.067$ & Beta & 11 \\
\hline Herpes oticus & 0.002 & $0-0.005$ & Beta & 11 \\
\hline Monaural deafness, given herpes oticus & 0.069 & $0.013-0.12$ & Beta & 11 \\
\hline \multicolumn{4}{|l|}{ Hospitalization given HZ } & 16 \\
\hline Age $60-69$ & 0.013 & $0.005-0.021$ & Beta & \\
\hline Age $70-79$ & 0.018 & $0.011-0.026$ & & \\
\hline Age $\geq 80$ & 0.055 & $0.042-0.068$ & $\S$ & \\
\hline \multicolumn{4}{|l|}{ Death due to HZ, per $1,000,000$ cases } & 17 \\
\hline Age $60-69$ & 2.22 & $1.72-2.72$ & Beta & \\
\hline Age $70-79$ & 6.18 & $5.32-7.03$ & & \\
\hline Age $80-89$ & 23.96 & $21.88-26.03$ & $\S$ & \\
\hline Age $\geq 90$ & 152.13 & $143.76-160.5$ & $\S$ & \\
\hline Duration of $\mathrm{HZ}$ hospitalization, mean days & 4.7 & $4.4-4.9$ & Normal & 18 \\
\hline Compliance rate & 0.6 & $0-1.0$ & Normal & 19 \\
\hline \multicolumn{4}{|l|}{ Vaccine efficacy against $\mathrm{HZ}$ incidence $(\%)$} & $4,5,7$ \\
\hline Initial efficacy at vaccination & 64.8 & $56.8-72.7$ & Normal & \\
\hline Annual waning rate & 5.4 & $3.7-7.2$ & Normal & \\
\hline \multicolumn{4}{|l|}{ Likelihood ratio, by age } & 20 \\
\hline Age $60-64$ & 1.797 & NA & NA & \\
\hline Age $65-69$ & 1.582 & NA & NA & \\
\hline Age $70-74$ & 0.742 & NA & NA & \\
\hline Age $75-79$ & 0.541 & NA & NA & \\
\hline Age $80-84$ & 0.232 & NA & NA & \\
\hline Age $\geq 85$ & 0.080 & NA & NA & \\
\hline \multicolumn{4}{|l|}{ Vaccine efficacy against BOI (\%) } & 4 \\
\hline Initial efficacy at vaccination & 70.8 & $57.6-84.0$ & Normal & \\
\hline \multirow{2}{*}{\multicolumn{4}{|c|}{$\begin{array}{l}\text { Annual wanıng rate } \\
\text { Vaccine efficacy against PHN incidence }(\%)\end{array}$}} & \\
\hline & & & & 4 \\
\hline Efficacy for the first 5 years & 63.2 & $42.2-76.5$ & Normal & \\
\hline Annual waning rate from year 6 & 10.0 & NA & NA & \\
\hline \multicolumn{4}{|l|}{ Adverse effect } & 5 \\
\hline Local reaction & 0.34 & $0.33-0.36$ & Beta & \\
\hline Serious reaction & 0.007 & $0.001-0.013$ & Beta & \\
\hline \multicolumn{5}{|l|}{ Utility } \\
\hline Monocular blindness & 0.92 & $0.885-0.948$ & Beta & 11 \\
\hline Monaural deafness & 0.97 & $0.958-0.982$ & Beta & 11 \\
\hline Post herpetic neuralgia after 6 months & 0.67 & $0.618-0.722$ & Beta & 21,22 \\
\hline Short term morbidities (QALYs) & & & & \\
\hline Acute herpes zoster & & & & 5,23 \\
\hline Age $60-69$ & 0.0129 & $0.0049-0.0207$ & Gamma & \\
\hline Age $\geq 70$ & 0.0216 & $0.0144-0.0286$ & & \\
\hline Hospitalization & 0.0129 & $0.012-0.013$ & Gamma & Length of stay \\
\hline Local reaction & 0.0001 & $0-0.0003$ & Gamma & Assumption \\
\hline Serious reaction & 0.0082 & $0.003-0.016$ & Gamma & \\
\hline Direct medical costs, $\$$ per case & & & & \\
\hline Acute HZ & 387 & $298-568$ & Gamma & 11 \\
\hline PHN & 762 & $651-906$ & Gamma & 11 \\
\hline Ophthalmic complications & 15,158 & $11,118-17,852$ & Gamma & 11 \\
\hline Herpes oticus & 481 & $135-943$ & Gamma & 11 \\
\hline Hospitalization for $\mathrm{HZ}$ & 7977 & $7477-8235$ & Gamma & 18 \\
\hline Serious reaction & 6609 & $6442-6703$ & Gamma & 18 \\
\hline
\end{tabular}


Table 1.. (continued)

\begin{tabular}{|c|c|c|c|c|}
\hline Variable & Baseline value & $\begin{array}{l}\text { Range for one-way } \\
\text { sensitivity analysis }\end{array}$ & PSA Distribution & Reference \\
\hline Indirect cost, $\$$ per $\mathrm{HZ}$ case & & & & 9 \\
\hline Age $60-64$ & 4678 & $3373-5982$ & Gamma & \\
\hline Age $\geq 65$ & 4409 & $3179-5639$ & & \\
\hline Vaccine price $^{*}$ & 187.89 & $120-300$ & NA & 24 \\
\hline Vaccine administration costs & 25 & $15-35$ & NA & 25 \\
\hline
\end{tabular}

*Although vaccine price is variable, it is determined by the manufacturer and not uncertain. Therefore, we did not define a probabilistic distribution for vaccine price 26

†Ranges for one-way sensitivity analysis generally represent $95 \%$ confidence intervals, except for costs, where wider ranges were tested $\neq$ Because the compliance rate is unknown, the range is from 0 to 1

$\S$ For model inputs with age-specific values, the distribution was first defined for the lowest age group, which was considered as the reference. Distributions for remaining age groups were determined by multiplying relative likelihood ratios among these ages and the reference age by the reference distribution. Because the value was drawn randomly from the distribution in PSA, this definition of distributions ensured that the probabilistic values of different age groups had the appropriate relative magnitudes compared with one another as when they were deterministic. ${ }^{9}$

HZ herpes zoster, PHN postherpetic neuralgia, BOI burden-of-illness, QALY quality-adjusted life year, PSA probabilistic sensitivity analysis, NA not applicable

strategies described above. For all strategies, people began in the "Healthy" state and moved to other health states according to transition probabilities (Table 1). Following each HZ episode, the patient could die, fully recover or experience complications.

Compared to the unvaccinated group, vaccinated patients had a reduction in disease incidence and complications proportional to vaccine efficacy, which waned over time. Following a booster, vaccine efficacy increased and then waned at the same rate as following initial vaccination. Model inputs were derived from the medical literature. Model outputs, including costs (vaccine and disease treatment costs) and outcomes (the number of HZ cases, PHN cases, and quality-adjusted life years or QALYs), were computed for each strategy. Based on the mid-range of the World Health Organization recommendation, ${ }^{27}$ we chose $\$ 100,000 / \mathrm{QALY}$ as the costeffectiveness threshold. The study was conducted from the societal perspective. Costs and QALYs were discounted at $3 \%$. Costs were expressed in 2014 US dollars. The medical care component of the Consumer Price Index was used to adjust for inflation. ${ }^{28}$ We developed the model in TreeAge Pro 2014 (TreeAge Software, Williamstown, MA).

\section{Model Inputs and Assumptions}

Estimates and ranges for the base-case and sensitivity analyses are presented in Table 1. Important inputs are described below. Epidemiologic Parameters. The incidence of $\mathrm{HZ}$ began rising before varicella vaccine was introduced. ${ }^{1,12,29}$ It could represent better reporting, lack of natural boosting from exposure to chicken pox, or other factors. To estimate this increasing rate, we fit several linear regressions using the sex-specific incidences for people aged $\geq 65$ during 1993-2006, as reported in the most comprehensive observational study of increasing HZ incidence. ${ }^{12}$ We then took the age- and sex-specific incidence from the largest HZ study in the U.S. ${ }^{13}$ as the baseline and applied the regression slopes to project incidences for 2010 (the latest year of rising incidence). We assumed incidence then remained stable because varicella vaccination has been implemented universally for 15 years $^{30}$ and advertising for Zostavax had raised general awareness. Moreover, our projected incidence for 2010 exceeded carefully adjudicated incidence among unvaccinated controls from 2010-2014. ${ }^{31}$

Age-specific incidence of $\mathrm{PHN}$, defined as $\mathrm{HZ}$ pain persisting $\geq 3$ months, was based on the Shingles Prevention Study (SPS). ${ }^{5}$ Age-specific HZ hospitalization rates were modeled on a large managed care organization. ${ }^{16} \mathrm{HZ}$ age-specific mortality rates were drawn from the CDC. ${ }^{17}$ Estimates and derivations of other complications have been described previously. ${ }^{11}$ We used the 2010 US life tables to derive background age- and sex-specific mortality. ${ }^{32}$ The sex distribution reflected the 2013 US population. ${ }^{33}$

Vaccine-Related Parameters. The SPS, a randomized trial of the live attenuated $\mathrm{HZ}$ vaccine, reported efficacy up to 4 years post-vaccination. ${ }^{5}$ After its completion, a subset of participants was re-enrolled for the Short-term Persistence Substudy (STPS), ${ }^{7}$ which reported efficacy up to 7 years postvaccination. The Long-term Persistence Substudy (LTPS) reported efficacy from 7 to 11 years post-vaccination. ${ }^{4}$

The SPS, STPS, and LTPS reported three efficacy end points: BOI, PHN incidence, and $\mathrm{HZ}$ incidence. ${ }^{4,}{ }^{5,7}$ BOI measured total pain from $\mathrm{HZ}$ for 182 days after disease onset. Efficacy for BOI was reported for the whole population, most of whom had a BOI of zero. It therefore incorporated the vaccine's impact on $\mathrm{HZ}$ incidence, as well as severity of pain from either $\mathrm{HZ}$ or PHN in the first 6 months. Efficacy against HZ and PHN incidence was also reported for the entire population. In order to separate these different vaccine effects, we first estimated the efficacy for $\mathrm{HZ}$ incidence by year since vaccination. We then estimated the additional efficacies for BOI and PHN incidence among $\mathrm{HZ}$ cases by developing an equation to describe the relationship between the efficacy for HZ incidence and the overall efficacy for BOI or PHN incidence of the entire population (see online Appendix). The efficacy function for $\mathrm{HZ}$ incidence was: $y=-0.0544 \times$ year +0.6478 , for BOI: $y=$ 
- $0.0437 \times$ year 0.7083 , and for PHN incidence: $y-0.1 \times$ year +1.218 (online Appendix Figure 2). Initial efficacy against $\mathrm{HZ}$ incidence was further adjusted for vaccination age using age-specific likelihood ratios calculated from the SPS. $^{20}$

A recent study showed that a booster administered after $\geq$ 10 years had similar safety and immunogenicity as a first dose among people $\geq 70$ years. ${ }^{10}$ We assumed that a booster dose would have the same efficacy as an initial dose administered at that age.

Compliance with multi-dose vaccine schedules varies by vaccine, but is consistently low among all age groups. ${ }^{34-36} \mathrm{In}$ our base-case, we assumed $60 \%$ compliance (i.e., the percentage of patients who would receive a booster), based on older adults' compliance with tetanus boosters. ${ }^{19}$

QALYS. Utility estimates have been described previously. ${ }^{11}$ QALY loss due to $\mathrm{HZ}$ was estimated using the average BOI scores from SPS. The scores were transformed into utilities based on a study that reported both BOI scores and EuroQOL5 D utilities. ${ }^{23}$ The utility of PHN beyond 6 months was based on the work of Edmunds et al. ${ }^{21}$ We assumed serious vaccine reactions represented allergic reactions and resulted in hospitalization with a utility of zero. Length of stay was based on hospitalizations for allergic reactions. ${ }^{18}$ Utilities were adjusted for age. ${ }^{37}$

Costs. We derived costs from various sources. We used the CDC's private sector vaccine price. ${ }^{24}$ Vaccine administration was based on the Medicare rate. ${ }^{25}$ Serious reactions were assumed to cost the same as allergic reactions requiring hospitalization. ${ }^{18}$ Local reactions were minimal and assumed not to incur costs. Direct medical costs for treatment of acute $\mathrm{HZ}, \mathrm{PHN}$, ocular complications, and herpes oticus were based on our previous study. ${ }^{11}$ Productivity loss due to $\mathrm{HZ}$ was estimated as described previously, ${ }^{9}$ and age-adjusted to reflect the percentage of people in the labor force. ${ }^{38}$ The 2012 Health Care Utilization Project was used to estimate the length of stay and hospitalization costs (ICD-9-CM codes 053.0-053.9 for $\mathrm{HZ}$ and 995.27 for allergic reactions). ${ }^{18}$

\section{Sensitivity Analysis}

One-Way Sensitivity Analysis. We conducted one-way sensitivity analysis to examine the effect of all epidemiologic, vaccine-related and utility parameters on the cost-effectiveness of vaccination strategies.

Two-Way Sensitivity Analysis. We conducted two-way sensitivity analysis by varying the initial efficacy against $\mathrm{HZ}$ incidence and its annual waning rate, and vaccine cost against compliance rate. Finally, we varied vaccine cost and the initial efficacy against $\mathrm{HZ}$ incidence.

Probabilistic Sensitivity Analysis. We employed 10,000 iterations of Monte Carlo simulation simultaneous varying all model inputs. We displayed the results as a costeffectiveness acceptability curve showing the percentage of iterations that each strategy had an ICER below a specific willingness-to-pay (WTP) threshold.

\section{Model Validation}

We validated our model by summing the annual cases of $\mathrm{HZ}$ and $\mathrm{PHN}$, and BOI scores in the vaccinated and non-vaccinated groups, and then calculating the vaccine efficacy for time periods corresponding to the SPS, STPS, and LTPS. ${ }^{4,5} 7$ Due to the lack of detailed information on sex distribution by age of SPS participants, we applied the overall sex distribution equally across age groups and estimated efficacy for people aged 60 and 70 years separately. We then weighted the efficacy by age distribution for $60-69$ and $\geq 70$ years to reflect that of the SPS.

\section{RESULTS}

\section{Model Validation}

Modeled efficacies for HZ incidence, PHN incidence, and BOI were very close (within 1-3 percentage points) to those reported in the trials (online Appendix Table 1).

Table 2. Discounted Costs and Quality-Adjusted Life Years (QALYs) of Different Vaccination Strategies

\begin{tabular}{|c|c|c|c|c|c|}
\hline Strategy & Total costs $(\$)$ & Incremental costs $(\$)$ & QALYs & Incremental QALYs & ICER (\$/QALY) \\
\hline No vaccine & 517 & & 12.8635 & & N/A \\
\hline Vaccine at 80 & 597 & & 12.8657 & & Extended dominance ${ }^{*}$ \\
\hline Vaccine at 60 & 646 & & 12.8657 & & Dominated $^{\dagger}$ \\
\hline Vaccine at 75 & 622 & & 12.8669 & & Extended dominance ${ }^{*}$ \\
\hline Vaccine at 65 & 648 & & 12.8669 & & Dominated $^{\dagger}$ \\
\hline Vaccine at 75 plus one booster dose & 653 & & 12.8675 & & Dominated $^{\dagger}$ \\
\hline Vaccine at 70 & 642 & 125 & 12.8677 & 0.0042 & 29,832 \\
\hline Vaccine at 60 plus one booster dose & 721 & & 12.8685 & & Dominated $^{\dagger}$ \\
\hline Vaccine at 70 plus one booster dose & 690 & 48 & 12.8690 & 0.0013 & 36,648 \\
\hline Vaccine at 65 plus one booster dose & 711 & & 12.8691 & & Extended dominance ${ }^{*}$ \\
\hline Vaccine at 60 plus 2 booster doses & 750 & 60 & 12.8694 & 0.0004 & 153,734 \\
\hline
\end{tabular}

*This strategy has higher ICER than the next more effective strategy

+ This strategy has higher cost than the next more effective strategy

ICER Incremental cost-effectiveness ratio 


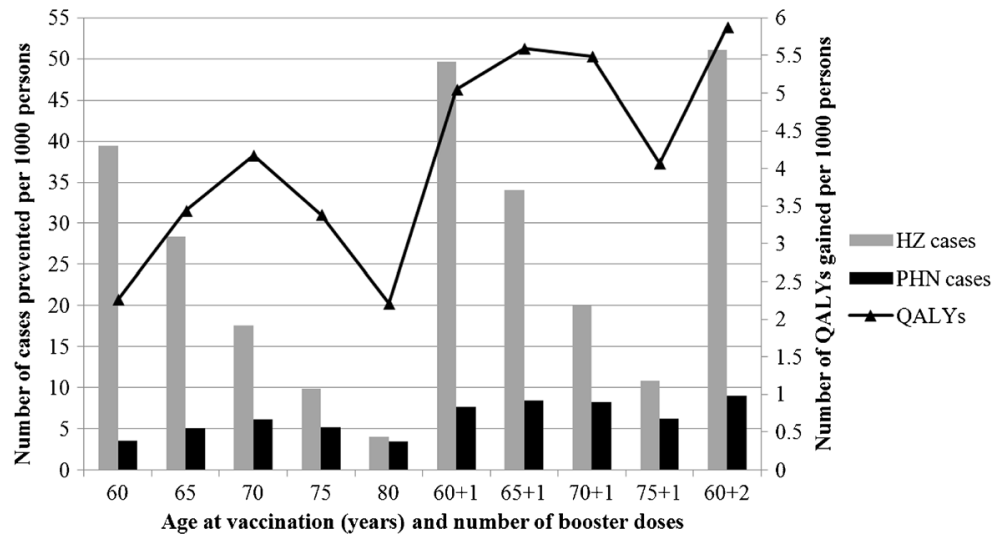

Figure 1. Clinical effectiveness of different vaccination strategies compared to no vaccination. Adding a booster was always more effective, i.e., preventing more cases and gaining more QALYs, than one-time vaccination in the same age. "Vaccination at 60 plus two boosters" was the most effective strategy, preventing the most number of cases and producing the highest number of QALYs. HZ, herpes zoster; PHN, postherpetic neuralgia; QALY, quality-adjusted life year.

\section{Base-Case Analysis}

Table 2 depicts the costs and QALYs of different vaccination strategies. All but four strategies were dominated. No vaccination had the lowest cost and produced the fewest QALYs. "Vaccination at 60 plus two boosters" provided the greatest number of QALYs, but with an ICER of $\$ 153,734 /$ QALY. At a willingness to pay of $\$ 100,000 / Q A L Y$, "vaccination at 70 years plus one booster" provided the most QALYs and was highly cost-effective with an ICER of \$36,648/QALY.

Figure 1 shows the number of $\mathrm{HZ}$ and $\mathrm{PHN}$ cases prevented and QALYs gained per 1000 vaccinated persons for each strategy compared to no vaccination. If no booster was available, "vaccination at 70" prevented more cases and produced more QALYs. Adding a booster after 10 years was always more effective than one-time vaccination at the same age. Finally, "vaccination at 60 plus two boosters" prevented the most cases and produced the most QALYs.

\section{Compliance rate (100\%-53\%) \\ Probability of $\mathrm{PHN} \geq 12$ months \\ QALY loss due to acute $\mathrm{HZ}^{*}$ \\ Vaccine price $(\$ 120-\$ 290)$}

Efficacy against $\mathrm{HZ}$ incidence at vaccination (72.7\%-56.8\%)

Waning rate of efficacy against $\mathrm{HZ}$ incidence $(3.7 \%-7.2 \%)$

Probability of $\mathrm{PHN}$ given $\mathrm{HZ}^{*}$

Probability of serious reactions (0.001-0.013)

Efficacy against PHN incidence in first 5 years $(42.2 \%-76.5 \%)$

Indirect costs.*

Probability of any ophthalmic complications (0.032-0.012)

\section{Sensitivity Analysis}

One-Way Sensitivity Analysis. Within the plausible parameter ranges for all variables, the four non-dominated strategies and their ordering did not change. Figure 2 displays all inputs that changed the ICER of "vaccination at 60 plus two boosters" by $>10 \%$. "Vaccination at 60 plus two boosters" would be the most cost-effective strategy at $\$ 100,000 / \mathrm{QALY}$ if compliance rate was $>67 \%$, QALYs lost due to HZ was equal to the lower bound of the $95 \% \mathrm{CI}$, vaccine cost was $<\$ 156 /$ dose, initial vaccine efficacy against $\mathrm{HZ}$ incidence was $>69.2 \%$, waning rate of efficacy against $\mathrm{HZ}$ incidence was $<4.5 \%$ year, or probability of having a serious vaccine reaction was $<0.27 \%$.

Two-Way Sensitivity Analysis. We found that "vaccination at 60 plus two boosters" was preferred at a threshold of $\$ 100,000 /$ QALY if initial vaccine efficacy was high and the rate of decline was low (Fig. 3-a). At a higher compliance rate,

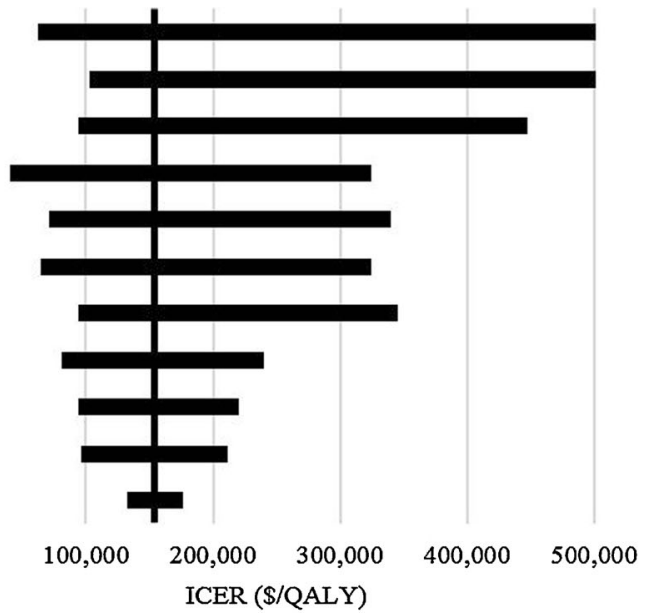

Figure 2. Tornado diagram of incremental cost-effectiveness ratio (ICER) of the "vaccination at 60 plus two booster doses" strategy. The diagram was limited at $\$ 500,000 / Q A L Y$ as the upper threshold because the strategy would be extremely non-cost-effective above that. The vertical line represents base-case ICER. HZ, herpes zoster; PHN, post-herpetic neuralgia; QALY, quality-adjusted life year. Note: * Ranges for these age-specific parameters are specified in Table 1, with the highest values leading to the leftmost ICERs. Lower or upper bounds of the ranges for all age groups were applied at the same time to maintain their age-specific relative magnitude. 
a

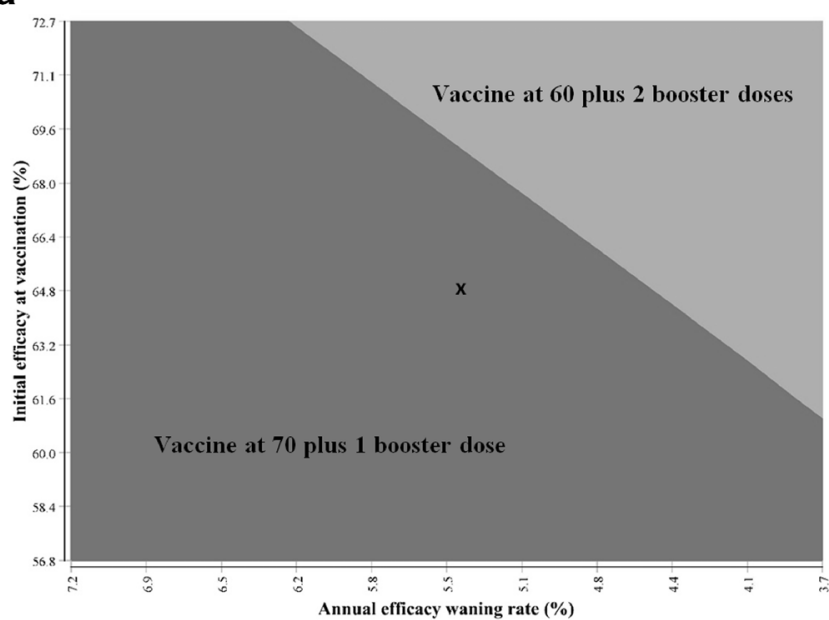

b

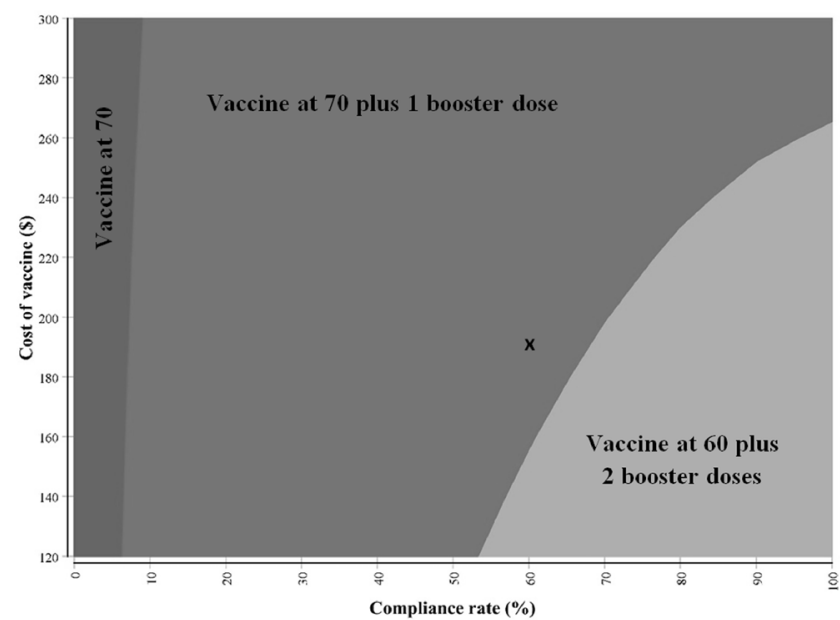

C

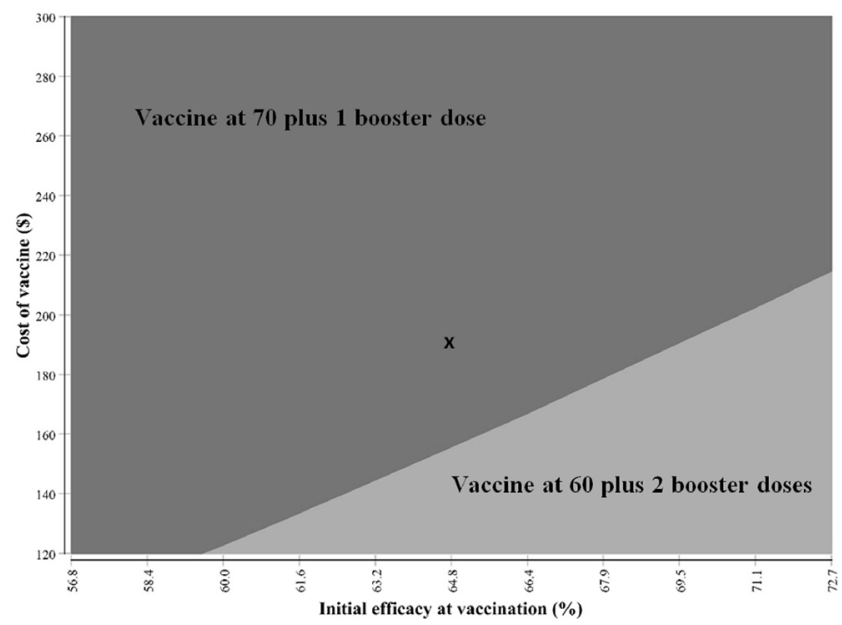

Figure 3. Two-way sensitivity analysis of impact of different combinations of variables using a willingness-to-pay threshold of $\$ 100,000 / q u a l i t y$-adjusted life year gained. Letter $\mathbf{X}$ denotes basecase. a Initial efficacy at vaccination and annual waning rate of the efficacy against herpes zoster incidence. b Vaccine cost and compliance rate. $\mathrm{c}$ Vaccine cost and the initial efficacy against herpes zoster incidence at vaccination.

"vaccination at 60 plus two boosters" was preferred unless the vaccine cost was high (Fig. 3-b). Finally, the lower the vaccine cost and the higher the initial efficacy against $\mathrm{HZ}$ incidence, the more cost-effective the "vaccination at 60 plus two boosters" was (Fig. 3-c).

Probabilistic Sensitivity Analysis. Figure 4 shows the probability of each strategy being preferred (i.e., offering the greatest number of QALYs) at different WTP thresholds. Between \$40,000/QALY - \$135,000/QALY, "vaccination at 70 plus one booster" was most likely to be preferred. At $\$ 100,000 /$ QALY threshold, "vaccination at 60 plus two boosters" had only $35 \%$ probability of being preferred.

\section{DISCUSSION}

Recent information about waning efficacy of $\mathrm{HZ}$ vaccine, as well as the safety and immunogenicity of a booster, raise questions regarding the optimal age to vaccinate and whether a booster dose is cost-effective. We used a decision analytic model that incorporated these new data to compare the lifetime benefit and cost-effectiveness of administering first-time vaccination and boosters at different ages. Assuming a WTP threshold of $\$ 100,000 / \mathrm{QALY}$, we found "vaccination at 70 plus one booster" to be the optimal immunization schedule. Vaccinating earlier prevented more cases of HZ, but avoided fewer cases of PHN due to a lower PHN incidence at younger ages. Because PHN has more impact on quality of life, later vaccination and boosting produced more QALYs. However, waiting too long produced fewer QALYs because patients were unprotected during high incidence years prior to vaccination.

Although "vaccination at 60 plus two boosters" prevented the most cases, it was very expensive and not cost-effective under base-case assumptions. However, if the vaccine were to cost $<\$ 156 /$ dose or if $>67 \%$ of patients received a booster dose, then "vaccination at 60 years plus two boosters" would cost $<\$ 100,000 /$ QALY. Using our best estimates for these values, and varying all model inputs simultaneously, "vaccination at 60 plus two boosters" had only a $35 \%$ chance of being cost-effective. "Vaccination at 70 plus one booster" was always the most likely to be cost-effective within the WTP range of $\$ 40,000 /$ QALY and $\$ 135,000 /$ QALY.

In agreement with previous analyses, ${ }^{39-42}$ our study confirmed that if a one-time vaccination (as currently recommended) were being considered, vaccination at age 70 would be optimal. Moreover, our study highlights the unintended consequence of offering one-time vaccination at age 60 . Because of waning immunity, patients vaccinated at age 60 receive $42 \%$ less lifetime protection against PHN than those vaccinated at 70 .

In 2015, a new adjuvanted subunit $\mathrm{HZ}$ vaccine (HZ/su) was demonstrated to prevent $97 \%$ of $\mathrm{HZ}$ cases over 3 years. ${ }^{31}$ Although the duration of protection is unknown, the licensing 


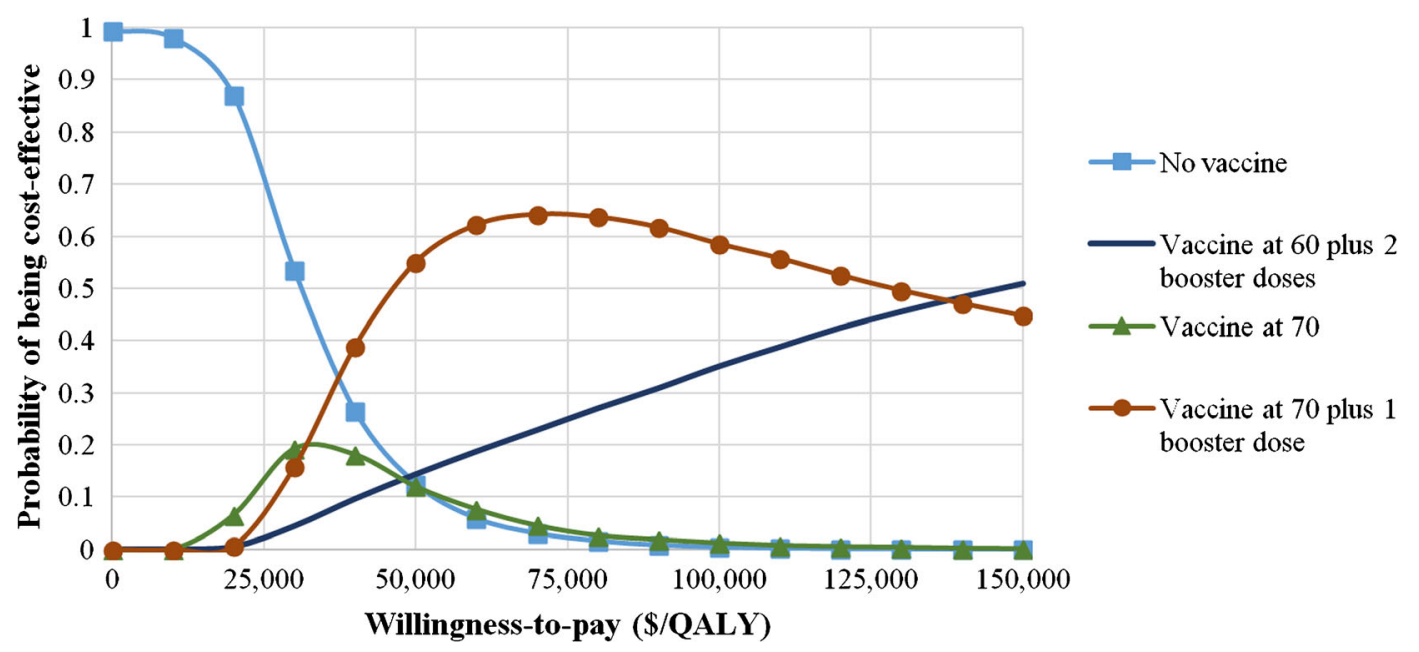

Figure 4. Cost-effectiveness acceptability curve of different vaccination strategies. Only strategies with $>5 \%$ the probability of being costeffective at different willingness-to-pay values were included. QALY, quality-adjusted life year.

of $\mathrm{HZ} / \mathrm{su}$ vaccine (expected in late 2016) should change the $\mathrm{HZ}$ vaccination paradigm. Based on our sensitivity analysis, given the new vaccine's efficacy, "vaccination at 60 plus two boosters" would be the optimal strategy if the vaccine cost $\leq$ $\$ 388$ for the two-dose regimen. However, until the vaccine is licensed, the cost will not be known. In addition, $96 \%$ of patients in the randomized trial received both doses. ${ }^{31}$ Other two-dose series, such as hepatitis A vaccine, have completion rates closer to $65 \%{ }^{34}$ Thus the new vaccine's effectiveness may be substantially lower than in the trial, and vaccination beginning at 70 years may still be preferred.

Our study has limitations. First, several variables relating to the booster are unknown. While it is reasonable to assume the booster will cost the same as initial vaccination, it will be years before we determine the booster's efficacy and compliance rate. Second, efficacy of HZ vaccine was based on the LTPS, an observational study using historical controls. ${ }^{4}$ Additional long-term trials are unlikely. Third, we assumed that the incidence of HZ stopped rising after 2010 because we could not assume an unlimited upward trajectory. Carefully adjudicated events from the recent vaccine study suggest our rates may be too high, ${ }^{31}$ but even a continued increase in incidence would not change the optimal vaccine schedule. Finally, we lacked contemporary estimates of costs associated with $\mathrm{HZ}$ and PHN; consequently, we inflated previous estimates to 2014 dollars, but our findings were insensitive to these costs.

Our study appears to be the first to incorporate trial data regarding long-term efficacy and persistence ${ }^{4}$ and the first U.S. study to address cost-effectiveness of vaccination strategies that include boosters. Our findings have important implications. The current ACIP recommendation is to administer one dose of vaccine to patients aged $\geq 60$ years. However, because efficacy beyond 10 years is limited, vaccination at age 60 appears to be one of the most expensive and least effective strategies. Based on our model, delaying vaccination by 10 years should reduce costs while improving outcomes. Adding a booster dose after 10 years should offer additional gains at reasonable cost. For patients who can afford it, beginning vaccination at age 60 followed by two booster doses 10 years apart would be most effective, but at a price which cannot be universally advocated. One potential exception would be if the compliance with the booster exceeded $67 \%$, then beginning vaccination at age 60 could be considered cost-effective. However, older adults' compliance with a tetanus booster, which also has a 10 -year interval, remains at $60 \%{ }^{19}$

In light of the data on waning efficacy, the ACIP may want to reconsider their recommendation. For patients already vaccinated, a booster appears to be in order. However, future vaccination efforts will likely have to be divided between delivering the initial vaccination and encouraging patients to get boosters. Following approval of $\mathrm{HZ}$ vaccine, uptake has been slow, hampered by high cost, vaccine shortages and lack of familiarity. ${ }^{43}, 44$ Although vaccination rates have begun to rise, ${ }^{19}$ the impact of a booster recommendation on vaccine supply and initial vaccination rates is unknown. One way to mitigate between these competing needs would be to focus on vaccinating all patients above age 70 , whether they are unvaccinated or have been vaccinated at least 10 years ago. By simply not vaccinating patients in their 60 s, physicians would ensure that all remaining strategies were cost-effective. Following several years of widespread booster use, we should be able to determine the booster's effectiveness and compliance rate. We will also have data on the cost and effectiveness of the adjuvanted subunit vaccine. At that time, it may be appropriate to revisit the recommendations. In the interim, current recommendations appear to be the least effective, and unnecessarily expensive.

Acknowledgments: The study was conducted without external funding. The manuscript was presented at the Society of General Internal Medicine Annual Meeting, held on May 11-14, 2016 in Hollywood, FL. 
Corresponding Author: Phuc Le, PhD, MPH; Center for Value-Based Care Research, Medicine InstituteCleveland Clinic, Cleveland, $\mathrm{OH}$ USA (e-mail: lep@ccf.org).

\section{Compliance with Ethical Standards:}

Conflict of Interest: The authors declare that they do not have a conflict of interest.

\section{REFERENCES}

1. Yawn BP, Saddier P, Wollan PC, St Sauver JL, Kurland MJ, Sy LS. A population-based study of the incidence and complication rates of herpes zoster before zoster vaccine introduction. Mayo Clin Proc. 2007;82(11):1341-9.

2. Kawai K, Gebremeskel BG, Acosta CJ. Systematic review of incidence and complications of herpes zoster: towards a global perspective. BMJ Open. 2014;4(6), e004833.

3. Jackson JL, Gibbons R, Meyer G, Inouye L. The effect of treating herpes zoster with oral acyclovir in preventing postherpetic neuralgia. A metaanalysis. Arch Intern Med. 1997;157(8):909-12.

4. Morrison VA, Johnson GR, Schmader KE, Levin MJ, Zhang JH, Looney DJ, et al. Long-term persistence of zoster vaccine efficacy. Clin Infect Dis. 2015;60(6):900-9.

5. Oxman MN, Levin MJ, Johnson GR, Schmader KE, Straus SE, Gelb LD, et al. A vaccine to prevent herpes zoster and postherpetic neuralgia in older adults. N Engl J Med. 2005;352(22):2271-84.

6. Schmader KE, Levin MJ, Gnann JW Jr, McNeil SA, Vesikari T, Betts RF, et al. Efficacy, safety, and tolerability of herpes zoster vaccine in persons aged 50-59 years. Clin Infect Dis. 2012;54(7):922-8.

7. Schmader KE, Oxman MN, Levin MJ, Johnson G, Zhang JH, Betts R, et al. Persistence of the efficacy of zoster vaccine in the shingles prevention study and the short-term persistence substudy. Clin Infect Dis. 2012;55(10): 1320-8.

8. Hales CM, Harpaz R, Ortega-Sanchez I, Bialek SR. Update on recommendations for use of herpes zoster vaccine. MMWR Morb Mortal Wkly Rep. 2014;63(33):729-31.

9. Le $\mathbf{P}$, Rothberg MB. Cost-effectiveness of herpes zoster vaccine for persons aged 50 years. Ann Intern Med. 2015;163(7):489-97.

10. Levin MJ, Schmader KE, Pang L, Williams-Diaz A, Zerbe G, Canniff J, et al. Administration of a second dose of herpes zoster vaccine ten years after a first dose. J Infect Dis. 2015.

11. Rothberg MB, Virapongse A, Smith KJ. Cost-effectiveness of a vaccine to prevent herpes zoster and postherpetic neuralgia in older adults. Clin Infect Dis. 2007;44(10):1280-8.

12. Leung J, Harpaz R, Molinari NA, Jumaan A, Zhou F. Herpes zoster incidence among insured persons in the United States, 1993-2006: evaluation of impact of varicella vaccination. Clin Infect Dis. 2011;52(3):332-40.

13. Insinga RP, Itzler RF, Pellissier JM, Saddier P, Nikas AA. The incidence of herpes zoster in a United States administrative database. J Gen Intern Med. 2005;20(8):748-53.

14. Bouhassira D, Chassany O, Gaillat J, Hanslik T, Launay O, Mann C, et al. Patient perspective on herpes zoster and its complications: an observational prospective study in patients aged over 50 years in general practice. Pain. 2012;153(2):342-9.

15. Helgason S, Petursson G, Gudmundsson S, Sigurdsson JA. Prevalence of postherpetic neuralgia after a first episode of herpes zoster: prospective study with long term follow up. BMJ. 2000;321(7264):794-6.

16. Jackson LA, Reynolds MA, Harpaz R. Hospitalizations to treat herpes zoster in older adults: causes and validated rates. Clin Infect Dis. 2008;47(6):754-9.

17. Centers for Disease Control and Prevention, National Center for Health Statistics. Compressed Mortality File 1999-2012 on CDC WONDER Online Database, released October 2014. Data are from the Compressed Mortality File 1999-2012 Series 20 No. 2R, 2014, as compiled from data provided by the 57 vital statistics jurisdictions through the Vital Statistics Cooperative Program. Available at: http://wonder.cdc. gov/cmf-icd10.html. Accessed 19 July 2016.

18. Agency for Healthcare Research and Quality. Healthcare cost and utilization project-HCUP. Available at http://hcupnet.ahrq.gov/HCUPnet.jsp Accessed 19 July 2016
19. Williams WW, Lu PJ, O'Halloran A, Bridges CB, Kim DK, Pilishvili T, et al. Vaccination coverage among adults, excluding influenza vaccination - United States, 2013. MMWR Morb Mortal Wkly Rep. 2015;64(4):95102

20. Zoster vaccine live (Oka/Merck) Zostavax. FDA clinical briefing document for Merck and Co., 2005 [Internet]. Available at: http://www.fda.gov/ ohrms/dockets/ac/05/briefing/5-4198b2_1.pdf. Accessed on 19 July 2016.

21. Edmunds WJ, Brisson M, Rose JD. The epidemiology of herpes zoster and potential cost-effectiveness of vaccination in England and Wales. Vaccine. 2001;19(23-24):3076-90.

22. Hornberger J, Robertus $\mathbf{K}$. Cost-effectiveness of a vaccine to prevent herpes zoster and postherpetic neuralgia in older adults. Ann Intern Med. 2006; 145(5):317-25.

23. Coplan PM, Schmader K, Nikas A, Chan IS, Choo P, Levin MJ, et al. Development of a measure of the burden of pain due to herpes zoster and postherpetic neuralgia for prevention trials: adaptation of the brief pain inventory. J Pain. 2004;5(6):344-56.

24. Centers for Disease Control and Prevention. Adult vaccine price list. Available at: http://www.cdc.gov/vaccines/programs/vfc/awardees/vaccine-management/price-list/. Accessed 29 July 2016.

25. Centers for Medicare and Medicaid services. Physician Fee Schedule. Available at: http://www.cms.gov/apps/physician-fee-schedule/search/search-results. aspx?Y=0\&T=0\&HT=0\&CT=3\&H1=90471\&M=5. Accessed 19 July 2016.

26. Briggs AH, Goeree R, Blackhouse G, O'Brien BJ. Probabilistic analysis of cost-effectiveness models: choosing between treatment strategies for gastroesophageal reflux disease. Med Decis Making. 2002;22(4):290-308.

27. WHO-CHOICE. Choosing interventions that are cost-effective. [Internet]. Available at: http://www.who.int/choice/en. Accessed 19 July 2016.

28. Bureau of Labor Statistic. Medical care component of the Consumer Price Index-All urban consumers. Available at: http://data.bls.gov/cgi-bin/ surveymost. Accessed 19 July 2016.

29. Hales CM, Harpaz R, Joesoef MR, Bialek SR. Examination of links between herpes zoster incidence and childhood varicella vaccination. Ann Intern Med. 2013;159(11):739-45.

30. Marin M, Guris D, Chaves SS, Schmid S, Seward JF, Advisory Committee on Immunization Practices, Centers for Disease Control and Prevention (CDC). Prevention of varicella: recommendations of the Advisory Committee on Immunization Practices (ACIP). MMWR Recomm Rep. 2007;56(RR-4):1-40.

31. Lal H, Cunningham AL, Godeaux O, Chlibek R, Diez-Domingo J, Hwang SJ, et al. Efficacy of an adjuvanted herpes zoster subunit vaccine in older adults. N Engl J Med. 2015;372(22):2087-96.

32. Arias E. United States life tables, 2010. Natl Vital Stat Rep. 2014;63(7):1-63.

33. U.S. Census Bureau, Population Division. Annual Estimates of the Resident Population by Single Year of Age and Sex for the United States: April 1, 2010 to July 1, 2013. Release Date: June 2014. Available at: http://factfinder.census.gov/faces/tableservices/jsf/ pages/productview.xhtml?src=bkmk. Accessed 19 July 2016.

34. Nelson JC, Bittner RC, Bounds L, Zhao S, Baggs J, Donahue JG, et al. Compliance with multiple-dose vaccine schedules among older children, adolescents, and adults: results from a vaccine safety datalink study. Am J Public Health. 2009;99(Suppl 2):S389-97.

35. Luman ET, Shaw KM, Stokley SK. Compliance with vaccination recommendations for U.S. children. Am J Prev Med. 2008;34(6):463-70.

36. Widdice LE, Bernstein DI, Leonard AC, Marsolo KA, Kahn JA. Adherence to the HPV vaccine dosing intervals and factors associated with completion of 3 doses. Pediatrics. 2011;127(1):77-84.

37. Hanmer J, Lawrence WF, Anderson JP, Kaplan RM, Fryback DG. Report of nationally representative values for the noninstitutionalized US adult population for 7 health-related quality-of-life scores. Med Decis Making. 2006;26(4):391-400

38. Labor Force Statistics from the Current Population Survey-Employment status of the civilian noninstitutional population by age, sex, and race [Internet]. Available at: http://www.bls.gov/web/empsit/cpseea13.htm . Accessed 29 July 2016.

39. Pellissier JM, Brisson M, Levin MJ. Evaluation of the cost-effectiveness in the United States of a vaccine to prevent herpes zoster and postherpetic neuralgia in older adults. Vaccine. 2007;25(49):8326-37.

40. Ultsch B, Weidemann F, Reinhold T, Siedler A, Krause G, Wichmann O. Health economic evaluation of vaccination strategies for the prevention of 
herpes zoster and postherpetic neuralgia in Germany. BMC Health Serv Res. 2013;13:359.

41. Bilcke J, Marais C, Ogunjimi B, Willem L, Hens N, Beutels P. Costeffectiveness of vaccination against herpes zoster in adults aged over 60 years in Belgium. Vaccine. 2012;30(3):675-84.

42. Ortega-Sanchez IR. Decision and cost-effectiveness analyses of herpes zoster vaccination in adults 50 years of age and older [Presentation]. Atlanta, GA: US Department of Health and Human Services, CDC; 2013.
43. Hurley LP, Lindley MC, Harpaz R, Stokley S, Daley MF, Crane LA, et al. Barriers to the use of herpes zoster vaccine. Ann Intern Med. 2010;152(9):555-60.

44. Lu PJ, Euler GL, Jumaan AO, Harpaz R. Herpes zoster vaccination among adults aged 60 years or older in the United States, 2007: uptake of the first new vaccine to target seniors. Vaccine. 2009;27(6):882-7. 